\title{
Inhibitors of rhomboid proteases
}

\author{
Eliane V. Wolf ${ }^{1}$ and Steven H. L. Verhelst ${ }^{*, 2,3}$
}

${ }^{1}$ Chair for Chemistry of Biopolymers, Technische Universität München, Weihenstephaner Berg 3, 85354 Freising, Germany

${ }^{2}$ Leibniz Institute for Analytical Sciences ISAS, e.V., Otto-Hahn-Str. 6b, 44227 Dortmund, Germany

${ }^{3}$ Laboratory of Chemical Biology, Department of Cellular and Molecular Medicine, University of Leuven, Herestr. 49, 3000 Leuven, Belgium

* To whom correspondence should be addressed: steven.verhelst@ kuleuven.be; tel +49 231 1392-4236 


\begin{abstract}
(300 words max)
Rhomboid proteases form the most widespread family of intramembrane proteases. They utilize a catalytic serine-histidine dyad located several $\AA$ below the surface of the membrane for substrate hydrolysis. Multiple studies have implicated rhomboid proteases in biologically and medically relevant processes. Several assays have been developed that are able to monitor rhomboid activity. With the aid of these assays, different types of inhibitors have been found, all based on electrophiles that covalently react with the active site machinery. Although the currently available inhibitors have limited selectivity and moderate potency, they can function as research tools and as starting point for the development of activity-based probes, which are reagents that can specifically detect active rhomboid species. Structural studies on complexes of inhibitors with the E. coli rhomboid GlpG have provided insight into how substrate recognition may occur. Future synthetic efforts, aided by high-throughput screening or structurebased design, may lead to more potent and selective inhibitors for this interesting family of proteases.
\end{abstract}

\title{
Graphical abstract
}

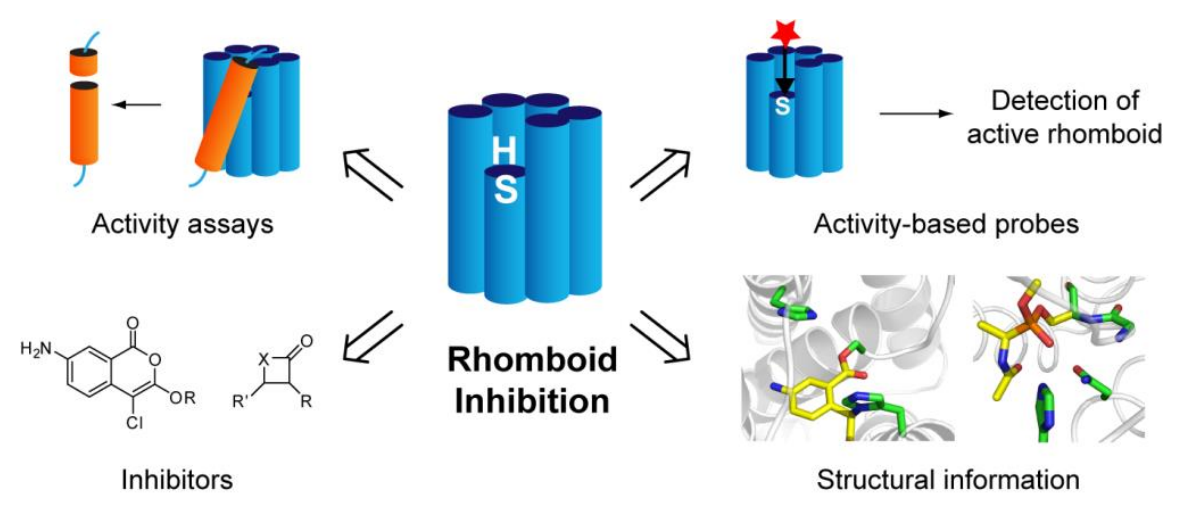




\title{
Highlights
}

- Rhomboid proteases are the most widespread intramembrane proteases

- Several different assays have been developed to monitor rhomboid protease activity

- Current inhibitor classes include different types of electrophiles

- Current inhibitors have limited potency and selectivity

- Crystal structures of rhomboid-inhibitor complexes provide mechanistic insights

Keywords activity-based probes, inhibitors, intramembrane proteases, rhomboids

\begin{abstract}
Abbreviations
ABP: activity-based probe, BODIPY: boron-dipyrromethylene, DCI: 3,4-dichloro-isocoumarin, DFP: diisopropyl-fluorophosphate, DmRho1: Drosophila melanogaster rhomboid-1, FITC: fluorescein isothiocyanate, IMP: intramembrane protease, MALDI-TOF: matrix-assisted laser desorption ionization time-of-flight, S2P: site-2 protease, SPP: signal peptide peptidase, TGF $\alpha$ : transforming growth factor alpha, TM: transmembrane helix.
\end{abstract}




\section{Introduction}

In 1997, site-2 protease was discovered as a first intramembrane protease (IMP) [1]. Although proteolysis within the membrane seems a counterintuitive process, the following years brought several other reports about IMPs. As our understanding of intramembrane proteolysis grows each year, IMPs have now turned from surprising exceptions to recognized members of the protease family [2-6].

From the primary amino acid sequence it is apparent that IMPs have an entirely different architecture compared with soluble proteases. However, their active sites resemble those of soluble proteases - a typical example of convergent evolution. IMPs contain multiple transmembrane helices (TMs) and reside within the lipid bilayer of a membrane. Their active sites are buried several $\AA$ below the surface of the membrane boundary and are formed by residues in different TMs (Fig. 1). The native substrates of IMPs are also transmembrane proteins and are cleaved within a TM or a juxtamembrane region. Through this cleavage, a soluble part of the substrate is released from the membrane to the exterior or interior of the cell or organelle, and can function in downstream pathways, in a role distinct from the full-length progenitor form [2-5]. IMPs can be classified into four groups: (1) Aspartyl IMPs, also GXGD proteases, named after part of their active site motif. Examples include the signal peptide peptidase family (SPP) [7] and $\gamma$-secretase [8, 9]; (2) glutamate IMPs, which include the recently discovered Rce1 [10]; (3) metallo IMPs, exemplified by the site-2 protease family (S2P) [1]; and (3) serine IMPs, also known as rhomboid proteases [11].

Rhomboids were first discovered in Drosophila melanogaster. In this organism, Rhomboid-1 cleaves the transforming growth factor $\alpha(\mathrm{TGF} \alpha)$ homolog Spitz, an important player in the epidermal growth factor receptor pathway $[11,12]$. Aided by bioinformatics, it became clear that rhomboids occur in almost

all organisms and are present in all phylogenetic domains of life: archaea, bacteria and eukaryota [13, 14]. A variety of studies have now indicated a functional role for rhomboids, for example in bacterial communication (quorum sensing) [15], cleavage of adhesins that apicomplexan parasites use to invade host cells [16-19], mitochondrial membrane dynamics [20-23], and cleavage of Pink-1, a protein involved in Parkinson's disease [24, 25].

In the past, the development of small molecule inhibitors and activity-based probes (ABPs) has been crucial for the study of soluble proteases. An advantage over genetic knock-outs is that small molecules can be introduced at a specific time and place. In addition, in the case of ABPs, the targeted proteases can be subsequently analyzed by biochemical methods. Because of their roles in biomedically relevant processes, rhomboid proteases could become future drug targets, but whether rhomboids are druggable, has not yet been validated. The development of selective inhibitors and other chemical tools for functional characterization of rhomboids will be crucial in order to answer this question. 
In this review article we will give an overview of the major milestones in rhomboid inhibitor research. We will first discuss the available activity assays that enable monitoring of rhomboid protease activity. Next, we will present the available inhibitors and discuss their respective merits. We will provide a brief overview of the available ABPs for the study of rhomboids. Finally, we will discuss crystal structures of rhomboids in complex with their inhibitors.

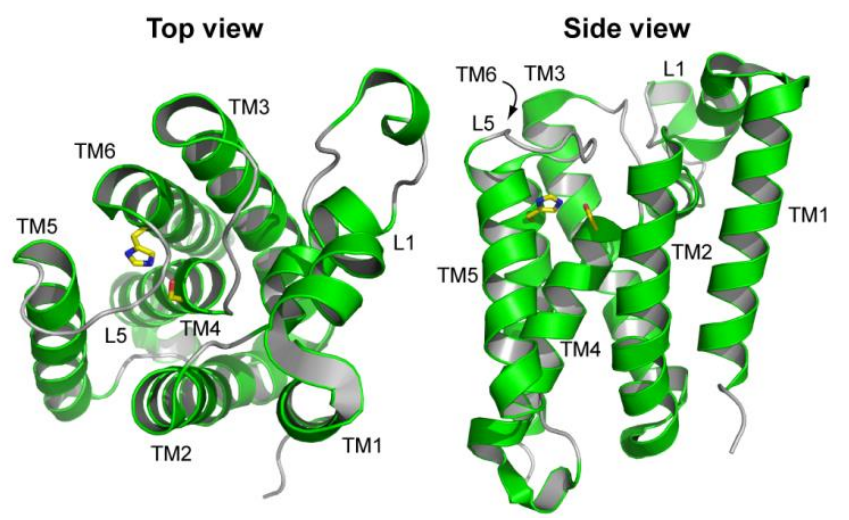

Fig. 1. The Escherichia coli rhomboid GlpG was the first IMP that was structurally characterized by Xray crystallography. Its structure is formed by six TMs, connected by several loops. The larger loop L1 is partially immersed within the membrane. Loop L5 sits as a 'cap' on top of the active site. S201 in TM4 and H254 in TM6 (both in stick representation) form the catalytic center, which is buried within the plane of the membrane.

\section{Activity assays}

An assay to detect rhomboid protease activity is a prerequisite for measuring inhibition of rhomboids. Several types of activity assays have been developed. Initially, these aimed to prove that rhomboids indeed act as proteases and hydrolyze membrane proteins. Later, assays were adapted or developed in order to screen for inhibition. In the next sections, we have grouped the assays according to the type of molecules that are used as a read-out for activity: membrane proteins, soluble polypeptides or proteins that incorporate fluorophores, and activity-based probes (Fig. 2).

\subsection{Membrane proteins}

The very first proof that rhomboids are a class of intramembrane proteases came from cell biology studies on Drosophila melanogaster Rhomboid-1 (DmRho1) [11]. DmRho1 was known to play a role in EGF signaling, but the mechanism was unclear. Transient expression of DmRho-1, the TGF- $\alpha$ homolog Spitz and its chaperone Star in eukaryotic cell culture showed that DmRho-1 cleaves Spitz within its TM, 
thereby setting it free from the membrane [11]. The proteolytic processing was confirmed by Western blot detection of the GFP-tagged substrate Spitz in the supernatant of the cells.

In general, proteolytic processing leads to a reduction in size and therefore a shift in retention in acrylamide gels (Fig. 2A). This principle has been used in various other studies using recombinant rhomboid substrates that incorporate various tags such as $\mathrm{His}_{6}$ or GFP. The assay is easy to perform and flexible, as it can be carried out in different settings: it has been used in living E. coli cells with Western blot detection [26, 27], on purified rhomboids in micelles or liposomes with either Western blot detection [28-30], Coomassie staining [31], autoradiography using an in vitro translated S35-labeled substrate [3234], or direct gel scanning using a fluorescently-labeled substrate [35]. This fluorescently-labeled substrate was based on a recombinant form of TatA (a native substrate of Providencia stuartii rhomboid AarA) that had been modified with a fluorophore using a Sortase A labeling strategy [36]. The same TatA protein was modified with derivatives from cyan and yellow fluorescent protein on the $\mathrm{N}$ - and C-terminal side, respectively, yielding a recombinant FRET membrane protein substrate. It was subsequently used in real-time kinetics measurements of different bacterial rhomboid species [30].

A reduction in the size of a substrate protein can also be measured by mass spectrometry. Vosyka et al. developed a MALDI-TOF-based assay to monitor cleavage of a recombinantly expressed and purified rhomboid substrate by E. coli GlpG and P. stuartii AarA [37]. This method was used to screen a small selection of compounds in order to find new rhomboid inhibitors. The benefit of the assay was the parallel measurement of up to 384 samples on a single MALDI-TOF plate and the automatic detection of the substrate and product peak intensities, from which the percentage of activity in comparison to a control was calculated [37].

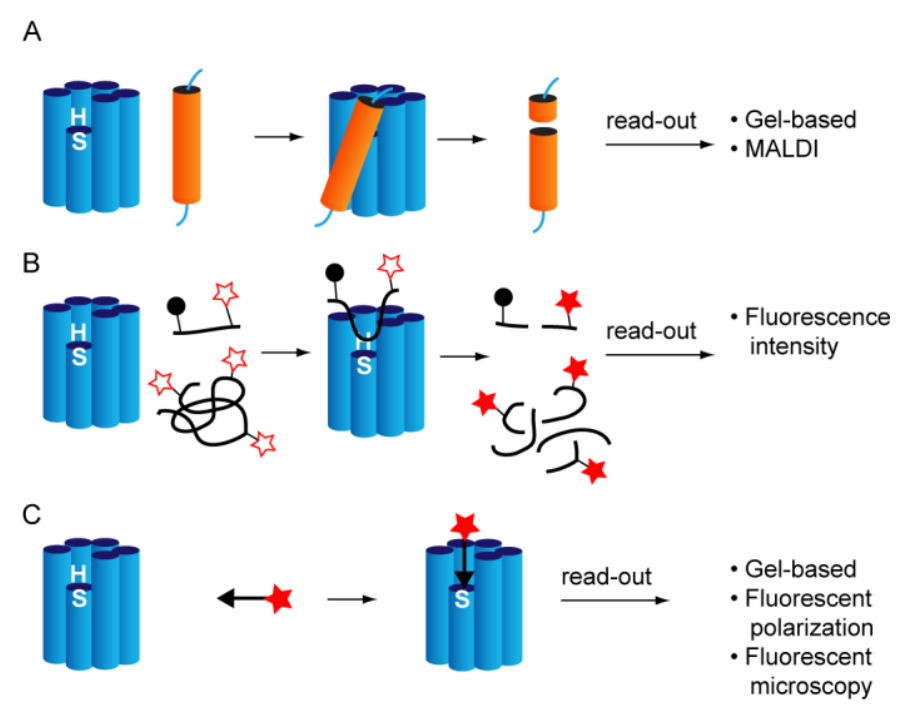


Fig. 2. Different methods to detect rhomboid protease activity. A) The cleavage of a transmembrane protein substrate within its TM results in protein fragments with a lower mass than the parent structure. The fragments can be analyzed by mass spectrometry or gel-based techniques. B) Surprisingly, soluble proteins or polypeptides can also be cleaved by rhomboid proteases. When decorated with multiple fluorophores or a fluorophore-quencher pair, this results in a fluorescently quenched reporter. Cleavage causes a gradual increase in fluorescence, which can be spectrophotometrically measured over time. C) Activity-based probes consist of a reactive group (triangle), a spacer (line) and a tag (star). Several ABPs have been reported for rhomboid proteases. They react with active rhomboid proteases and facilitate detection by gel-based or fluorescence techniques.

\subsection{Soluble fluorescent proteins or peptides}

Fluorescently-labeled casein (e.g. FITC-casein [38] or BODIPY-casein [39]) is a commercially available protein that has been used as a general substrate for a variety of proteases. Because multiple fluorophores are incorporated on the same protein molecule, the fluorescence is quenched (Fig. 2B). Therefore, these proteins cannot only be used in a gel-based read-out but also for spectrophotometric detection in real time.

The group of Akiyama showed that BODIPY-labeled casein can also be cleaved by rhomboid (GlpG) and its processing monitored in time by measurement of fluorescent intensity [26]. At the moment, it is not known at which sites casein is cut and how this proteolytic processing takes place, but it is likely that the soluble substrate approaches the catalytic machinery from the 'top' side when the L5 'cap' that covers the active site (Fig. 1) lifts upwards. The same substrate was used by the group of Ha to show that removal of the cytoplasmic domain of GlpG reduces the cleavage rate [40]. In a later study, BODIPYlabeled casein was applied in order to demonstrate that GlpG does not form a stable complex with the inhibitor DCI, but slowly regains activity over time [41]. The group of Lemieux applied BODIPY-casein in kinetic studies of rhomboid proteases. It was found that the cytoplasmic domain of GlpG does not have a profound effect on the catalytic parameters $k_{\text {cat }}$ and $K_{M}$ [42]. Furthermore - aided by experiments with a membrane protein substrate - it was found that some bacterial rhomboids are allosterically regulated and contain an exosite that is formed upon dimerization [30].

The Freeman laboratory designed a synthetic 15-mer polypeptide, based on the sequence around the scissile bond of Gurken, a D. melanogaster TGF $\alpha$ homolog that is cleaved by rhomboids from several species. The peptide was decorated with a fluorophore on one end of the cleavage site and a fluorescence quencher on the other end [43]. This resulted in a fluorogenic substrate suitable for high throughput screening. A different type of 'quenched' substrate was discovered by the Urban laboratory. The substrate, based on the first 33 amino acids of TatA, was chemically synthesized with an N-terminal 
FITC. Surprisingly, when this peptide was incorporated into liposomes, the FITC fluorescence was quenched, and cleavage by GlpG resulted in an increase of fluorescence over time [44]. The substrate was applied in the study of rhomboid kinetics in a membrane environment.

\subsection{Activity-based probes}

ABPs (Fig. 2C) are often derived from covalent electrophilic inhibitors and incorporate a detection tag such as a biotin or a fluorophore. Since they only react with the active enzyme species, they can be utilized as a read-out of an enzyme's activity state. For rhomboids, several activity-based probes have been reported [35, 37, 45]. They enable a variety of different detection methods including gel-based analysis [35, 37, 45], fluorescent polarization in 96-well plate format [35], and fluorescent microscopy [46]. The structures and applications of rhomboid ABP will be discussed in more detail in section 4 .

\section{Inhibitors}

The discovery of rhomboid proteases in 2001 also marks the start of the search for rhomboid protease inhibitors. All currently known inhibitor classes are listed with numbers in Fig. 3. Experiments with DmRho-1 suggested that rhomboids are insensitive to most broad-spectrum serine protease inhibitors. Only high concentrations of 3,4-dichloroisocoumarin (DCI; 1 Fig. 3A) and $N$-tosyl-phenylalaninechloromethyl ketone (TPCK; 9) were able to block processing of the model substrate Spitz by DmRho-1 in cell culture experiments [11]. Inhibitors with other structures have also been reported - most of them for GlpG. They include isocoumarin analogs, fluorophosphonates, $\beta$-lactams, $\beta$-lactones and peptidyl chloromethyl ketones. All of these inhibitors display a covalent inhibition mechanism (Fig. 3): they consist of electrophiles that react with the active site serine and in some cases with an additional residue. In the next sections, we will discuss all currently known classes of rhomboid inhibitors. $\mathrm{IC}_{50}$ values of covalent inhibitors heavily depend on the time that the enzyme is preincubated before a read-out of activity is performed. It is therefore hard to compare the inhibitor potency from different studies. Where we mention potency, we therefore also indicate the preincubation time.

\subsection{Isocoumarin analogs}

As mentioned above, DCI was found as the first rhomboid inhibitor, blocking the activity of DmRho1 [11]. In later studies, DCI was found to inhibit rhomboids from a wide range of organisms: P. stuartii AarA [28], B. subtilis YqgP [28, 32], E. coli GlpG [41], the rhomboid from A. aeolicus [28], T. gondii ROM5 [18], H. sapiens PARL [47, 48] and RHBDL2 [49]. DCI is therefore considered a pan-rhomboid inhibitor. We must stress, however, that DCI is not selective for rhomboids, but inhibits a wide range of 
serine proteases, and that the potency of DCI varies considerably between rhomboids (Wolf \& Verhelst, unpublished observations).

In a series of experiments that aimed at making crystals of GlpG in complex with DCI, it was found that the covalent bond between the active site serine and the carbonyl group of DCI is not stable [41]. It is therefore not surprising that a crystal structure could not be obtained. GlpG turns over the DCI by slowly hydrolyzing the ester bond (Fig. 3A). Over the course of several days, it thereby regains its activity [41]. This is consistent with results obtained for soluble serine proteases [50].

JLK-6 (2), an isocoumarin analog with a 7-amino-group, was also found to inhibit GlpG [51]. Due to the presence of a 7-amino group, the reaction mechanism is different from DCI (Fig. 3B). After attack of the active site serine onto the isocoumarin carbonyl group, the scaffold opens and forms a secondary electrophile, which subsequently reacts with the active site histidine H254. This mechanism is known from soluble serine proteases, such as porcine pancreatic elastase [52]. For GlpG, the formation of a double-bonded enzyme-inhibitor complex was confirmed by X-ray crystallography [51] as well as by a gel-shift assay [37]. The gel-shift can be explained by a lower apparent molecular weight of the more compact crosslinked species. The ester bond formed between the serine and the carbonyl group of the isocoumarin slowly hydrolyzes over time, just like for DCI [37]. However, GlpG remains inactive due to the irreversible alkylation of the histidine residue.

The isocoumarin derivative S016 (3) was found to be one order of magnitude more potent than DCI ( $\mathrm{IC}_{50}$ of $0.7 \mu \mathrm{M}$ versus $6 \mu \mathrm{M} ; 30 \mathrm{~min}$ preincubation time) [37]. Interestingly, a different binding mode was observed for derivative S016 compared to JLK-6. Instead of reaction with active site histidine H254, it forms a covalent bond with H150, which is part of the oxyanion hole (see also paragraph 5).

\section{2 $\beta$-lactams and $\beta$-lactones}

In a high-throughput screen of almost 60,000 compounds against the rhomboid AarA, $N$-sulfonylated $\beta$ lactams (4) were identified as rhomboid inhibitors (Fig. 3C) [43]. The $N$-sulfonyl group can be replaced by a carbamate group (5) [43, 53]; both are electron withdrawing groups that activate the lactam electrophile. Upon reaction with the active site serine, the lactam ring opens, which was corroborated by X-ray crystallography (see paragraph 5) [53]. Most $\beta$-lactams that showed activity against AarA, were more potent against GlpG. The most potent compound displayed an $\mathrm{IC}_{50}$ value of $0.4 \mu \mathrm{M}$ against GlpG (30 min preincubation). A small number of $\beta$-lactams were also capable of inhibiting mouse rhomboid-2 [43].

Wolf et al. reported $\beta$-lactones (6) as modest inhibitors of $\mathrm{GlpG}\left(\mathrm{IC}_{50}\right.$ of $26-44 \mu \mathrm{M}$ with $30 \mathrm{~min}$ preincubation) [35]. $\beta$-lactones have a similar central scaffold as $\beta$-lactams and also open the four- 
membered ring upon reaction with the active site serine (Fig. 3C). The reported compounds contained a terminal alkyne functionality, which is amenable to functionalization by click chemistry (see paragraph 4). The covalent mechanism of these compounds could therefore be shown on gel after functionalization of the covalent enzyme-inhibitor complex with a fluorescent dye [35].

A
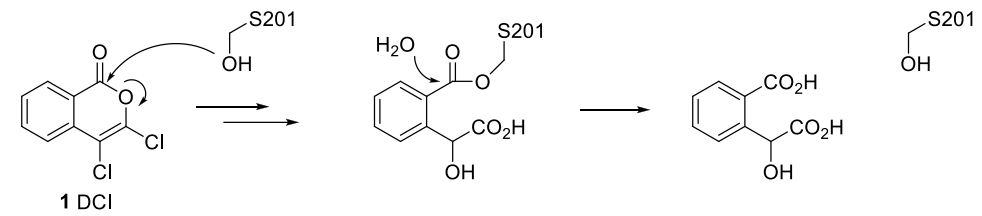

B

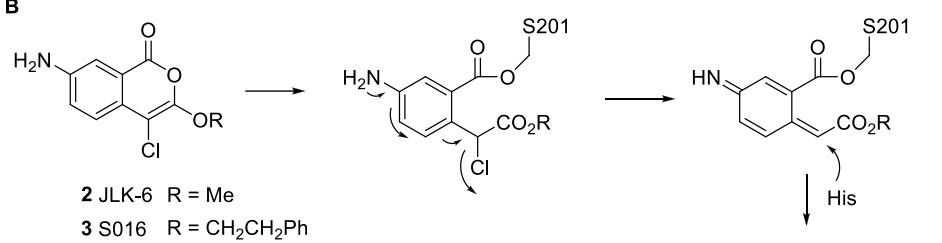

C

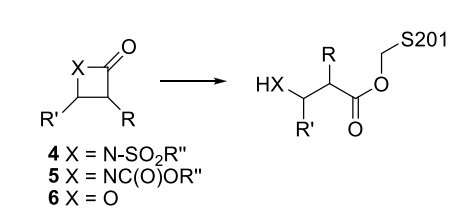

E
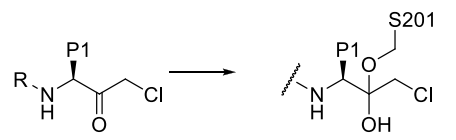

$9 \mathrm{R}=$ tosyl, $\mathrm{P} 1=\mathrm{Phe}$

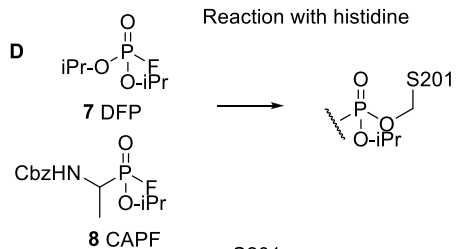

$10 \mathrm{R}=\mathrm{Ac}-\mathrm{IAT}, \mathrm{P} 1=\mathrm{Ala}$

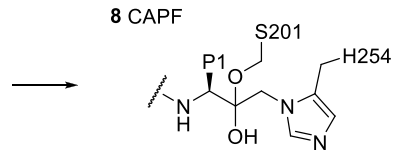

Fig. 3. Structures and mechanisms of all currently known classes of rhomboid protease inhibitors. A) DCI reacts with the active site serine of rhomboids, yielding an ester intermediate that slowly hydrolyzes. B) 4-chloro-isocoumarins such as JLK-6 and S016 carry a substituent at the 7-position of the isocoumarin scaffold. This results in the formation of a quinone imine methide electrophile, which can react with a histidine residue in the active site, forming a stable covalent enzyme-inhibitor complex. C) $\beta$-lactams and $\beta$-lactones react with the active site serine, opening the 4-membered ring structure. D) Fluorophosphate DFP or fluorophosphonate CAPF react at the electrophilic phosphoryl atom with the fluoride acting as a leaving group; $i \mathrm{Pr}=$ isopropyl. E) Chloromethyl ketones first form a reversible hemi-ketal with the active site serine, analogous to a tetrahedral intermediate. Next, the active site histidine is alkylated by attack onto the chloromethylene group.

\subsection{Others}

In 2012, the Ha laboratory showed that the general serine hydrolase inhibitor DFP (7) completely and irreversibly inhibits GlpG at concentrations $80 \mu \mathrm{M}$ by reaction with the active site serine. The more peptide-like fluorophoshonate CAPF (8), mimicking an alanine residue, also leads to a covalent adduct 
with the S201 of GlpG (Fig. 3D) [41]. The application in lysates or cells may be limited, since peptidyl fluorophosphonates are unstable in aqueous solution and rapidly hydrolyze [54].

The early observation that TPCK (9) is a weak inhibitor of DmRho-1, urged Zoll et al. to synthesize a number of peptidyl chloromethyl ketone analogs that have a longer peptide chain with residues that are based on the substrate specificity of GlpG in the P4-P1 position (10) [34]. These compounds react with GlpG by forming a hemi-ketal with the active site serine followed by alkylation of the active site histidine (Fig. 3E), as confirmed by X-ray crystallography (see paragraph 5). Although the resulting compounds were not very potent ( $\mathrm{IC}_{50}$ value of approximately $100 \mu \mathrm{M}, 180 \mathrm{~min}$ preincubation), they represent the first peptidic inhibitors that likely form similar interactions with the protease as a substrate.

\section{Activity-based probes}

ABPs are small molecule tools that can probe the activity of enzymes. Generally, they consist of a reactive group, a spacer, and a reporter tag. The reactive group often consists of an electrophilic trap that covalently binds to an active site residue in a mechanism-based reaction. The electrophilic trap is commonly derived from covalent enzyme inhibitors. The spacer may contain binding motifs for the enzyme that influence the selectivity of the ABP. The reporter tag, such as a biotin or a fluorophore, can be used for purification or detection of the probe-bound target. Efforts in synthetic chemistry have allowed to fine-tune the selectivity of ABPs, resulting in broad-spectrum probes for tagging an entire family of enzymes or highly selective probes that target only one single enzyme [55]. ABPs have been especially useful in protease research $[56,57]$.

In the past, fluorophosphonates (Fig. 4A) have been used as broad-spectrum ABPs for serine hydrolases $[58,59]$. They are based on the broad spectrum serine protease and esterase inhibitor DFP. Although DFP only acts as a weak rhomboid protease inhibitor, the fluorophosphonate ABPs react with the active site serine of GlpG at concentrations as low as $10 \mathrm{nM}$ [45]. More importantly, these ABPs react with rhomboid proteases from a wide variety of organisms (Wolf \& Verhelst, unpublished observation).

Sherratt et al. used the fluorescently labeled fluorophosphonate ABP FP-PEG-Rh (Fig. 4A) to identify residues in GlpG that are directly involved in the formation of an intact catalytic site [45]. Wolf et al. developed an inhibitor screening method for GlpG based on fluorescent polarization with a FP-Rh [35], a technique originally developed as a high-throughput screening method for poorly characterized soluble enzymes without the necessity of having a substrate [60]. This method is based on the large difference in molecular size of an unbound and an enzyme-bound ABP, resulting in a large difference in the tumbling rate. When the fluorophore is excited with polarized light, an unbound ABP will emit depolarized light, whereas the fluorescence of a bound ABP will remain polarized (Fig. 4B). It turned out to be crucial to have a suitable detergent that does not interfere with fluorescence polarization and also solubilizes 
rhomboid in an active form. Using this assay, $\beta$-lactones were found as novel rhomboid inhibitors [35]. The $\beta$-lactones inhibitors carried alkyne groups amenable to the introduction of a detection tag by click chemistry, which classifies these compounds as ABPs as well (Fig 4C). Vosyka et al. reported ABPs based on the isocoumarin inhibitor scaffold, all containing alkyne groups [37]. Experiments in this study demonstrated that these compounds can be used either in a two-step click chemistry labeling approach or directly as pre-clicked probes that already contain a fluorophore (Fig. 4D). Gel-based analysis also showed that the ester bond formed between the carbonyl group of the isocoumarin and the side chain of the active site serine residue is labile and hydrolyzes over time, but that alkylation of the histidine leads to a stable complex (Fig. 3B).

Recently, ABPs were also applied in the labeling of rhomboid proteases in liposomes [46]. In contrast to detergent micelles, liposomes embed rhomboids within a lipid bilayer. However, this study showed that the identification of inhibitors is not affected by the environment, confirming that micelle-solubilized rhomboids are a suitable model system. Furthermore, it was shown that fluorescent ABPs can be used to visualize rhomboids in giant unilamellar vesicles by fluorescence microscopy [46].
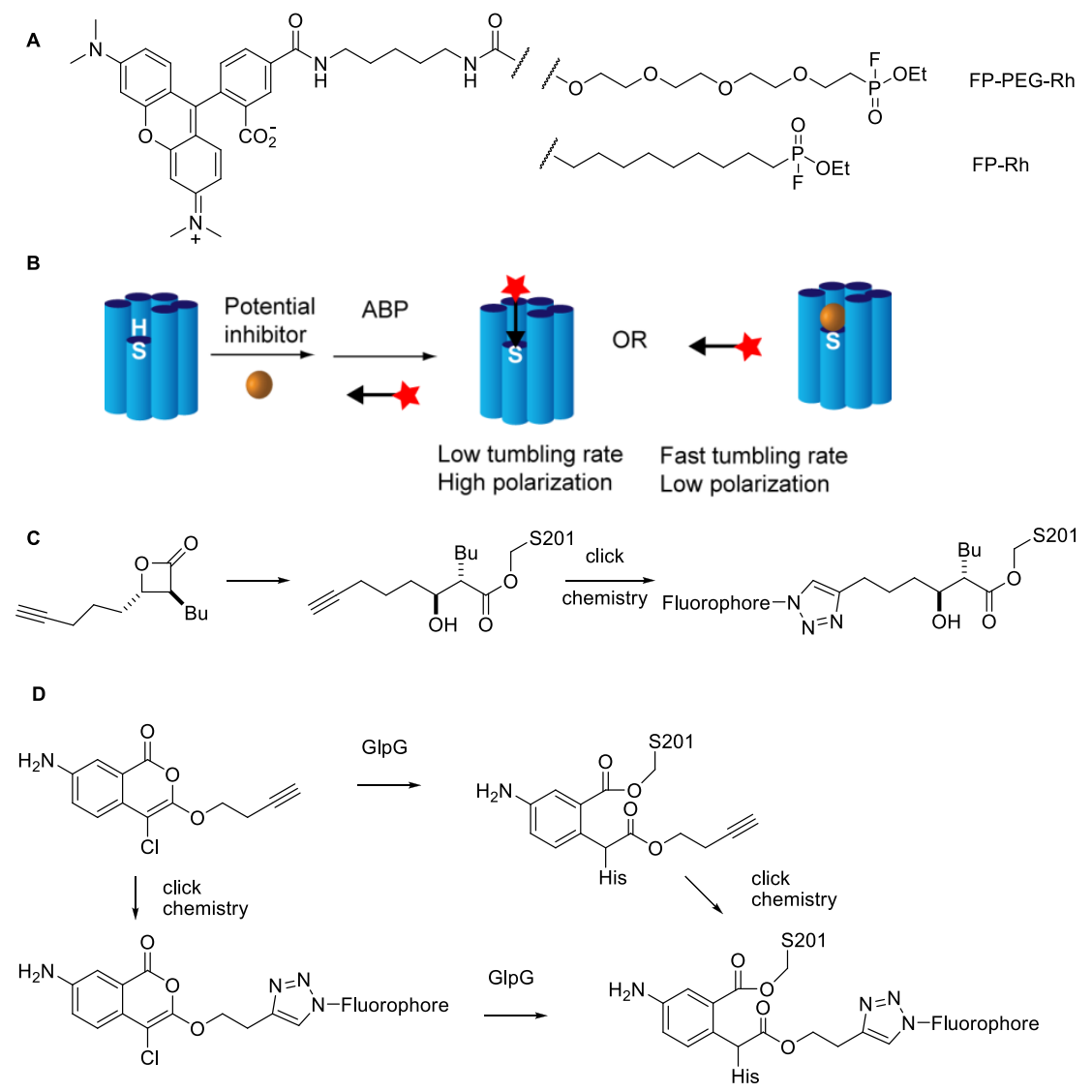

Fig. 4. Activity-based probes for rhomboid proteases. A) The fluorophosphonates FP-PEG-Rh and FP$\mathrm{Rh}$, containing a tetramethylrhodamine fluorophore, can both be utilized as rhomboid protease ABPs. 
Analogous to DFP, they react with the active site serine residue. B) Schematic representation of fluorescent polarization activity-based protein profiling for rhomboid proteases. A rhomboid-ABP complex has a low tumbling rate, which does not result in a loss of polarization of the emitted fluorescence. An inhibited rhomboid cannot react with the ABP. The unbound ABP has a high tumbling rate, which will result in depolarized fluorescence. C) $\beta$-lactone inhibitors with a terminal alkyne group can function as ABPs. The covalent complex of the rhomboid and the inhibitor can be decorated with a fluorophore or another detection tag by click chemistry. D) Alkyne-containing isocoumarins have also been applied as ABPs. They can either be used as is and tagged at level of the inhibitor-rhomboid complex or they can be pre-clicked with a fluorophore and used without requirement for further functionalization at the protein level.

\section{Inhibitor-rhomboid structures}

In 2007, Ha and co-workers reported the crystal structure of the E. coli rhomboid GlpG, which was the first structure of an intramembrane protease [40]. It was quickly followed by several other structures, of GlpG from E. coli [61, 62] and H. influenzae [63]. These structures showed that the rhomboid consists of six alpha-helical TMs creating a cup-like structure containing an active site Ser-His dyad for catalysis, which is located approximately $10 \AA$ below the membrane surface (Fig. 1). Several water molecules were found within the protein cavity, explaining how hydrolysis can take place within the membrane. The active site serine, located on top of the shorter TM4 helix, is surrounded by the other TMs, and in several structures, the active site is closed by a cap formed by loop 5 (L5). This implies that the substrate can only approach the active site machinery upon rearrangement of the rhomboid structure. The reported crystal structures led to several hypotheses about the mechanism: either through a lateral gate between TM2 and TM5 after movement of TM5 or by bending into the active site after lifting of cap L5 (Fig. 5A). The lateral gate model is supported by mutational analysis of the TM2/TM5 interface [27, 64]. However, a different study showed that chemical crosslinking of TM2 and TM5 did not have a significant effect on proteolysis [31]. Some recent studies, however, suggest again that widening of the space between TM2 and TM5 is possible and leads to a cleavage of the model substrate Spitz at a site that is located deeper into the membrane $[29,66]$.

All in all, it still remains elusive how substrates interact with rhomboids, since no co-crystal structure of a rhomboid with a substrate has been reported. Complexes of the E. coli rhomboid GlpG with different inhibitors, however, have provided insights into how substrates might be recognized. The first of these structures was solved by Vinothkumar et al. [51] and contains JLK-6 (2). From crystallographic studies of soluble serine proteases it was known that 4-chloro-isocoumarins with a 7-amino function may not only react with the active site serine, but also with a second nucleophile in the active site [52]. The GlpG JLK- 
6 structure indeed showed an opened isocoumarin ring structure and binding to both catalytic residues, S201 and H254 (Fig. 5B). The carbonyl group is within hydrogen bonding distance of several residues including H150 and N154, part of the oxyanion hole. Furthermore, the 7-aminogroup occupies a space which is believed to be the $S 1$ pocket.

Structures with several other inhibitors have also been elucidated: with DFP (7) [41], the phosphonate CAPF (8) [65], the sub-micromolar isocoumarin S016 (3) [37], several $N$-carbamoylated $\beta$-lactams (5) [53] and a tetrapeptide chloromethyl ketone (10) [34]. The similarities between the structures are striking and the several interesting observations can be made. Most structures show interaction of phosphoryl or carbonyl oxygen atom with residues in the oxyanion hole (Fig. 5C) $[34,37,41,51,65]$. Only in the $\beta$ lactam structures, the oxygen points away from these residues [53]. In all inhibitor-rhomboid complexes, loop L5 that covers the active site is partially lifted or disordered (Fig. 5D). This indicates that L5 needs to move to grant access to the active site. TM5 only shows subtle movement upon inhibitor binding, but it must be noted that the inhibitors are small compared to a full substrate, especially at the primed site.
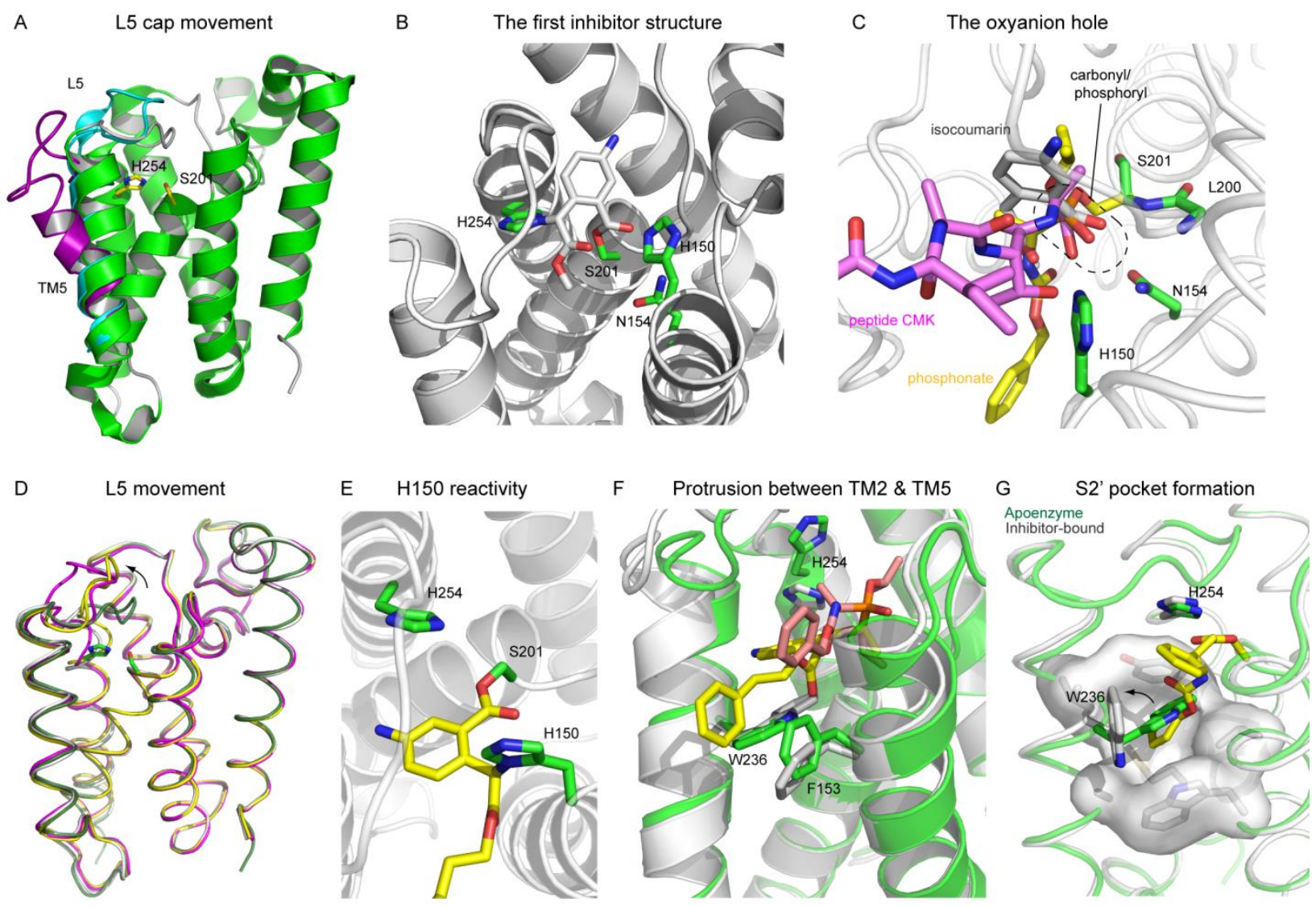

green: apoenzyme
white, yellow, magenta: inhibitor-bound

Fig. 5. Structural characterization of E. coli rhomboid GlpG aided by inhibitors. PDB codes are given for each structure. A) The L5 cap lifts upwards upon binding of most inhibitors (cyan - 3UBB). Apoenzyme is depicted in green (2IC8). One other apoenzyme did not only show lifting of the L5 cap, but also 
movement of TM5 (3NRF). B) A crystal structure of the isocoumarin JLK-6 with GlpG (2XOW) confirms that the inhibitor is bound to two amino acid side chains: S201 and H254. C) The oxyanion hole is formed by side chains of H150 and N154 and the backbone amides of L200 and S201. Note that the carbonyl of the isocoumarin (2XOW; inhibitor in white), the carbonyl of the peptidyl chloromethyl ketone (4QO2; inhibitor in magenta) and the phosphoryl group of CAPF (3UBB; inhibitor in yellow) all point towards these residues. D) An overlay of the apoenzyme (green; 2IC8) with three inhibitor-bound structures shows that there is virtually no movement in TM5, but that the L5 cap is lifted (yellow: 2XOW, magenta: 3UBB) or disordered (white: 3ZMI). E) The more potent isocoumarin S016 (in yellow) showed a different binding mode and reacted with H150 (3ZEB). F) Both the phenethyl group on the isocoumarin S016 (in yellow) and the Cbz group of CAPF (in pink) protrude between TM2 and TM5, above the residues F153 and W236. The GlpG structure bound to S016 is drawn in white, GlpG bound to CAPF is depicted in green. G) Plastic formation of the S2' pocket upon binding of a $\beta$-lactam inhibitor (3ZMI). The apoenzyme (2IC8) is depicted in green, the inhibitor-bound enzyme in white. The inhibitor, covalently bound to S201 is colored yellow. Rotation of the side chain of W236 opens a cavity formed by the side chains of residues W157, V204, Y205, M208, A233 and I237 (surface representation).

In contrast to the structure with JLK-6, isocoumarin S016 (3) shows a different binding mode: covalent binding occurs to S201 and the non-catalytic residue H150 (Fig. 5E). The reactivity of H150 suggests that this sidechain might have additional functions besides the stabilization of the oxyanion hole. The difference compared with JLK-6 may be explained by the hydrophobic interaction of the phenyl substituent that extends between TM2 and TM5 and may 'pull' the reactive electrophile away from H254. Interestingly, in the structure of CAPF (8) and $N$-carbamoylated $\beta$-lactams (5), a hydrophobic substituent also protrudes between TM2 and TM5 (Fig. 5F). It suggests that this could be the entry point for a substrate peptide chain to enter the active site. This idea is supported by recent molecular dynamics calculations [34].

In several of the GlpG-inhibitor structures, it was found that the pockets to accommodate the substituents on the inhibitors - and likewise the sidechains of a protein substrate - were plastically formed upon the interaction with the ligands. Binding of $\beta$-lactams, for example, led to rotation of W236 together with lifting of the L5 cap, opening the S2' pocket (Fig. 5G) [53]. A crystal structure of a tetrapeptide chloromethyl ketone, the most substrate-like structure to date, revealed that the S4 subsite adjusts itself depending on the P4 residue, by rearrangements of residues in the L1 loop [34].

At present, it is still ambiguous how a substrate precisely interacts with the rhomboid protease and gains access to the active site. The two different models comprise: (1) A lateral gate between TM2 and TM5, which controls the reaction kinetics (Fig. 6A, left). Substrates are discriminated from nonsubstrates 
through their intrinsically less stable helices [44]. (2) Access to the active site by a lifted loop L5 (Fig. $6 \mathrm{~A}$, right). Formation of substrate recognition pockets, such as the S2' pocket, takes place through subtle, binding-induced rearrangements (Fig. 6B). The helical substrate part that remains outside of the active site may bind to the rhomboid surface at a yet unidentified exosite, as first suggested by the Freeman group [33] (Fig. 6A, right).

We must emphasize that both models have several features in common. In both cases, a partially unwound substrate bends into the active site between TM2 and TM5. This either occurs above the residues W236 and F153, as suggested by crystal structures with inhibitors [37, 53, 65] and crosslinking studies [31], or the TM2-TM5 interface may be opened wider and grant a deeper access of the substrate into the active site $[27,64,66]$. In both models, displacement of the L5 loop facilitates binding of the nonprimed site substrate residues.

Upon the binding of the substrate, the scissile bond is able to approach the active site serine, located on top of the shorter helix of TM4. The serine oxygen nucleophile, activated by the active site histidine, now attacks the scissile bond. The resulting tetrahedral intermediate is stabilized through interactions with several residues in the oxyanion hole (Fig. 6B). Collapse of the tetrahedral intermediate gives an acylenzyme and an amine product corresponding to the primed site peptide. In the last steps, the acyl-enzyme is hydrolyzed with aid of a water molecule, which may be retained by a specific 'water retention site' [67]. This ends the catalytic cycle and forms back the active rhomboid species. 


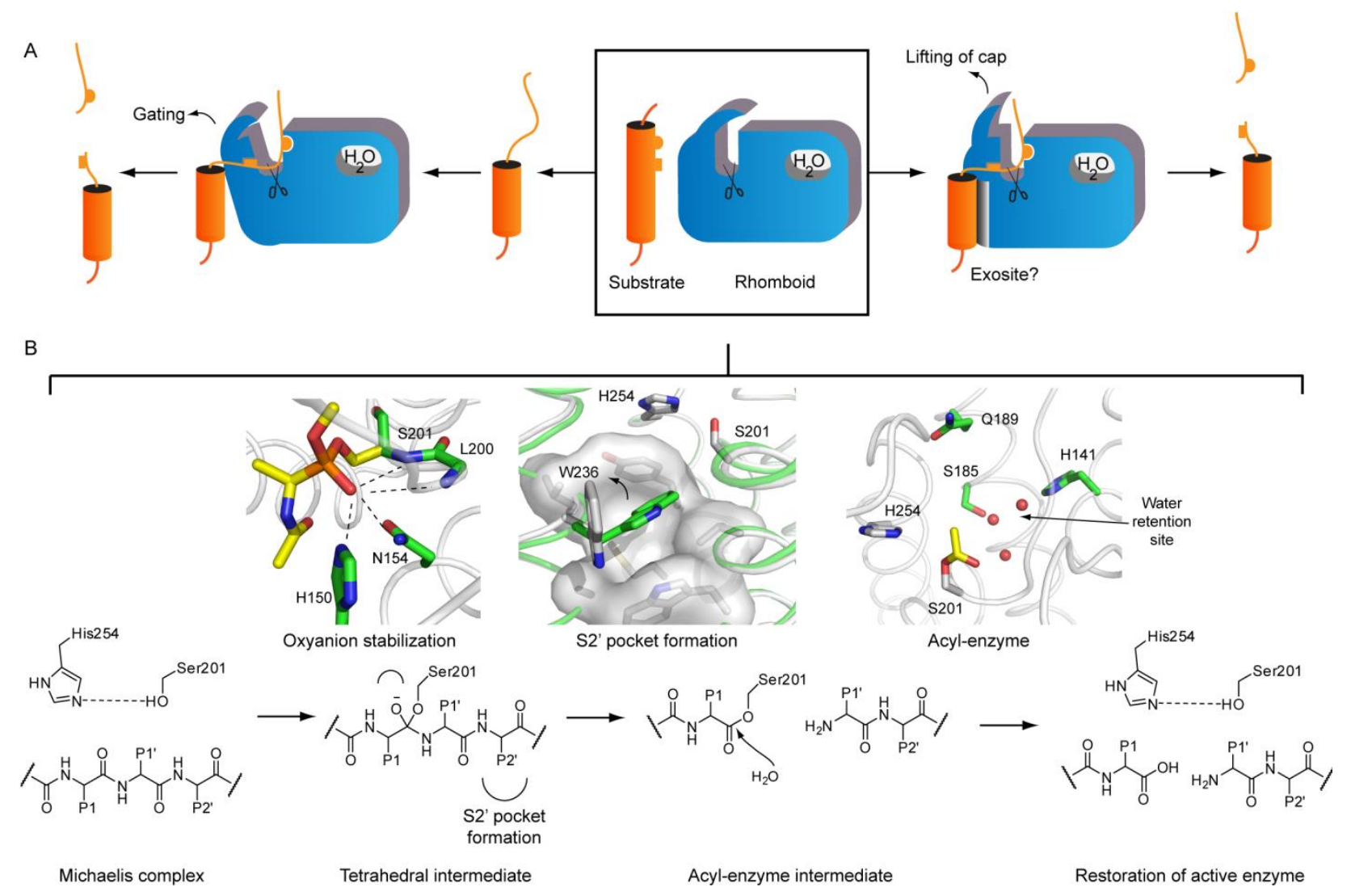

Fig. 6. A) Two models of substrate cleavage. Left: an intrinsically instable helix will unwind and enter the rhomboid active site between the lateral TM2/TM5 gate, upon which cleavage occurs. Right: lifting of cap L5, and binding to recognition pockets around the active site and possibly an exosite brings the substrate in contact with the active site machinery and cleavage takes place. B) Several close-ups of key features. Left: The catalytic serine attacks the scissile bond, leading to formation of the tetrahedral intermediate, which is stabilized by the oxyanion hole. Middle: amino acid side chains on the substrate interact with several binding-incuded pockets, such as the depicted S2' pocket, formed by movement of W236. Right: the acyl enzyme intermediate (only the carbonyl and $\mathrm{C} \alpha$ are depicted) undergoes hydrolysis with the aid of a water molecule, which may originate from a water retention site near the active site. Bottom scheme: chemical mechanism of substrate hydrolysis and restoration of the active enzyme.

\section{Conclusion and future directions}

Although rhomboid proteases are structurally the best characterized family of IMPs, the development of inhibitors is still in its infancy. In this review, we have discussed several aspects of rhomboid inhibitors, ranging from the available activity assays, to the reported inhibitors and ABPs, and the usage of inhibitors in structural studies. Although multiple substrates to measure rhomboid activity are known, a reporter substrate that functions for all rhomboids is still missing. Further knowledge of the substrate specificity of 
rhomboid proteases may yield such a substrate. To date, substrate specificity studies have focused on modifications in a few model substrates. The discovery of more native rhomboid substrates would therefore be beneficial, also in view of the further elucidation of the biological roles of rhomboids.

X-ray crystallography studies on covalent complexes of GlpG with different inhibitors have revealed a variety of different structural features, such as the location of the oxyanion hole, the plasticity of the pockets to which the inhibitor (and likely the substrate) binds, and the possible entry point of the substrate into the active site. However, continued future efforts in organic chemistry, biochemistry and structural biology are needed to draw a full picture of the rhomboid-substrate interaction.

The potency of most current rhomboid inhibitors is modest. In addition, their selectivity is not well studied. For some of these inhibitors, the selectivity is low, e.g. DFP and DCI, which are broad spectrum serine protease inhibitors. Also the $\beta$-lactones have been reported to react with various other targets [68]. For the reported $\beta$-lactams, isocoumarins and tetrapeptide chloromethyl ketones, the selectivity is not well defined. Some $\beta$-lactams showed good selectivity for GlpG over chymotrypsin with ratios of the apparent $\mathrm{IC}_{50}$ up to approximately 130 [43]. However, it is unknown whether they may react with other proteases or hydrolases. For isocoumarin S016, a more than 60-fold selectivity was found over trypsin, but it showed slightly higher activity against chymotrypsin [37]. The selectivity of the GlpG inhibitors based on tetrapeptide chloromethyl ketones is not reported, but it is known from previous studies that chloromethyl ketone electrophiles can react with both serine and cysteine proteases [54].

In view of the above, a major future challenge in rhomboid research is the development of potent inhibitors that display selectivity over other proteases and possibly over rhomboids from other organisms. But how can the potency and the selectivity of rhomboid inhibitors be increased? Peptidyl chloromethyl ketones are restricted to interaction with the non-primed site. Although the reported peptide scaffold already matches an optimal cleavage motif of GlpG [34], the usage of non-natural amino acids, as successfully applied for other types of proteases [69], may increase potency and selectivity. Some analogs of $\beta$-lactams and isocoumarins have been synthesized [37, 43], but additional optimization of the substituents in both scaffolds might result in higher selectivity. Since crystal structures are reported for all of the above mentioned inhibitor classes, a structure-based approach could be taken. However, one problem is that the recognition pockets around the active site seem to be formed upon binding of the inhibitor, which complicates the exploration of potential extra binding sites.

Several papers have mentioned the possible presence of an exosite on rhomboid proteases, first as a proposed model [33], and later supported by calculations [70] and experimental data [30, 71]. In addition, a ubiquitin interaction motif was found on the cytosolic domain of RHBDL4 [71]. These areas of the rhomboid protease could form extra interaction sites for inhibitors, and a combination of an active site binder with an exosite binder would likely provide higher selectivity and potency. However, such 
inhibitors will require additional structural information as well as considerable synthetic chemistry efforts.

Due to the roles of rhomboids in medically relevant processes, the future may bring validation of a rhomboid protease as a drug target. All reported inhibitors to date display a covalent inhibition mechanism. Although drug development programs tend to favor reversible inhibitors, recent years have seen an increase in attention for covalent binders $[72,73]$. It is perhaps interesting to note that compounds with $\beta$-lactam and $\beta$-lactone scaffolds are currently applied in the treatment of several diseases, such as bacterial infections and obesity. Both scaffolds may therefore form suitable starting points for future development of rhomboid targeting drugs, if selectivity and potency requirements can be met. Reversible inhibitors of rhomboid proteases have not yet been reported. One of the reasons may be the low affinity of rhomboids towards their substrates in comparison with other proteases [44]. As a consequence, the affinity towards substrate-inspired reversible inhibitors may also be low. Future efforts in high-throughput screening and structure-based design are required to confirm or contest this idea.

\section{Acknowledgements}

We thank the Deutsche Forschungsgemeinschaft (DFG) for funding.

\section{References}

[1] R.B. Rawson, N.G. Zelenski, D. Nijhawan, J. Ye, J. Sakai, M.T. Hasan, T.Y. Chang, M.S. Brown, J.L. Goldstein, Complementation cloning of S2P, a gene encoding a putative metalloprotease required for intramembrane cleavage of SREBPs, Mol Cell, 1 (1997) 47-57.

[2] M.S. Wolfe, R. Kopan, Intramembrane proteotysis: Theme and variations, Science, 305 (2004) 11191123.

[3] M.S. Wolfe, Intramembrane proteolysis, Chem Rev, 109 (2009) 1599-1612.

[4] M.K. Lemberg, Intramembrane proteolysis in regulated protein trafficking, Traffic, 12 (2011) 11091118.

[5] K. Strisovsky, Structural and mechanistic principles of intramembrane proteolysis--lessons from rhomboids, FEBS J, 280 (2013) 1579-1603.

[6] D. Langosch, C. Scharnagl, H. Steiner, M.K. Lemberg, Understanding intramembrane proteolysis: from protein dynamics to reaction kinetics, Trends Biochem Sci, (2015).

[7] A. Weihofen, K. Binns, M.K. Lemberg, K. Ashman, B. Martoglio, Identification of signal peptide peptidase, a presenilin-type aspartic protease, Science, 296 (2002) 2215-2218. 
[8] M.S. Wolfe, W. Xia, C.L. Moore, D.D. Leatherwood, B. Ostaszewski, T. Rahmati, I.O. Donkor, D.J. Selkoe, Peptidomimetic probes and molecular modeling suggest that Alzheimer's gamma-secretase is an intramembrane-cleaving aspartyl protease, Biochemistry, 38 (1999) 4720-4727.

[9] M.S. Wolfe, W. Xia, B.L. Ostaszewski, T.S. Diehl, W.T. Kimberly, D.J. Selkoe, Two transmembrane aspartates in presenilin-1 required for presenilin endoproteolysis and gamma-secretase activity, Nature, 398 (1999) 513-517.

[10] I. Manolaridis, K. Kulkarni, R.B. Dodd, S. Ogasawara, Z. Zhang, G. Bineva, N. O'Reilly, S.J. Hanrahan, A.J. Thompson, N. Cronin, S. Iwata, D. Barford, Mechanism of farnesylated CAAX protein processing by the intramembrane protease Rce1, Nature, 504 (2013) 301-305.

[11] S. Urban, J.R. Lee, M. Freeman, Drosophila Rhomboid-1 defines a family of putative intramembrane serine proteases, Cell, 107 (2001) 173-182.

[12] J.R. Lee, S. Urban, C.F. Garvey, M. Freeman, Regulated intracellular ligand transport and proteolysis control EGF signal activation in Drosophila, Cell, 107 (2001) 161-171.

[13] E.V. Koonin, K.S. Makarova, I.B. Rogozin, L. Davidovic, M.C. Letellier, L. Pellegrini, The rhomboids: a nearly ubiquitous family of intramembrane serine proteases that probably evolved by multiple ancient horizontal gene transfers, Genome Biol, 4 (2003) R19.

[14] M.K. Lemberg, M. Freeman, Functional and evolutionary implications of enhanced genomic analysis of rhomboid intramembrane proteases, Genome Res, 17 (2007) 1634-1646.

[15] L.G. Stevenson, K. Strisovsky, K.M. Clemmer, S. Bhatt, M. Freeman, P.N. Rather, Rhomboid protease AarA mediates quorum-sensing in Providencia stuartii by activating TatA of the twin-arginine translocase, Proc Natl Acad Sci USA, 104 (2007) 1003-1008.

[16] T.J. Dowse, J.C. Pascall, K.D. Brown, D. Soldati, Apicomplexan rhomboids have a potential role in microneme protein cleavage during host cell invasion, Int J Parasitol, 35 (2005) 747-756.

[17] S.A. Howell, F. Hackett, A.M. Jongco, C. Withers-Martinez, K. Kim, V.B. Carruthers, M.J. Blackman, Distinct mechanisms govern proteolytic shedding of a key invasion protein in apicomplexan pathogens, Mol Microbiol, 57 (2005) 1342-1356.

[18] F. Brossier, T.J. Jewett, L.D. Sibley, S. Urban, A spatially localized rhomboid protease cleaves cell surface adhesins essential for invasion by Toxoplasma, Proc Natl Acad Sci USA, 102 (2005) 4146-4151.

[19] R.P. Baker, R. Wijetilaka, S. Urban, Two Plasmodium rhomboid proteases preferentially cleave different adhesins implicated in all invasive stages of malaria, PLoS Pathog, 2 (2006) e113.

[20] G.A. McQuibban, S. Saurya, M. Freeman, Mitochondrial membrane remodelling regulated by a conserved rhomboid protease, Nature, 423 (2003) 537-541. 
[21] M. Herlan, F. Vogel, C. Bornhovd, W. Neupert, A.S. Reichert, Processing of Mgm1 by the rhomboid-type protease Pcp1 is required for maintenance of mitochondrial morphology and of mitochondrial DNA, J Biol Chem, 278 (2003) 27781-27788.

[22] H. Sesaki, S.M. Southard, A.E. Hobbs, R.E. Jensen, Cells lacking Pcp1p/Ugo2p, a rhomboid-like protease required for Mgm1p processing, lose mtDNA and mitochondrial structure in a Dnm1pdependent manner, but remain competent for mitochondrial fusion, Biochem Biophys Res Commun, 308 (2003) 276-283.

[23] S. Cipolat, T. Rudka, D. Hartmann, V. Costa, L. Serneels, K. Craessaerts, K. Metzger, C. Frezza, W. Annaert, L. D'Adamio, C. Derks, T. Dejaegere, L. Pellegrini, R. D'Hooge, L. Scorrano, B. De Strooper, Mitochondrial rhomboid PARL regulates cytochrome $\mathrm{c}$ release during apoptosis via OPA1-dependent cristae remodeling, Cell, 126 (2006) 163-175.

[24] E. Deas, H. Plun-Favreau, S. Gandhi, H. Desmond, S. Kjaer, S.H. Loh, A.E. Renton, R.J. Harvey, A.J. Whitworth, L.M. Martins, A.Y. Abramov, N.W. Wood, PINK1 cleavage at position A103 by the mitochondrial protease PARL, Hum Mol Genet, 20 (2011) 867-879.

[25] C. Meissner, H. Lorenz, A. Weihofen, D.J. Selkoe, M.K. Lemberg, The mitochondrial intramembrane protease PARL cleaves human Pink1 to regulate Pink1 trafficking, J Neurochem, 117 (2011) 856-867.

[26] S. Maegawa, K. Ito, Y. Akiyama, Proteolytic action of GlpG, a rhomboid protease in the Escherichia coli cytoplasmic membrane, Biochemistry, 44 (2005) 13543-13552.

[27] S. Urban, R.P. Baker, In vivo analysis reveals substrate-gating mutants of a rhomboid intramembrane protease display increased activity in living cells, Biol. Chem., 389 (2008) 1107-1115.

[28] S. Urban, M.S. Wolfe, Reconstitution of intramembrane proteolysis in vitro reveals that pure rhomboid is sufficient for catalysis and specificity, Proc. Natl. Acad. Sci. USA, 102 (2005) 1883-1888.

[29] S.M. Moin, S. Urban, Membrane immersion allows rhomboid proteases to achieve specificity by reading transmembrane segment dynamics, eLife, 1 (2012) e00173.

[30] E. Arutyunova, P. Panwar, P.M. Skiba, N. Gale, M.W. Mak, M.J. Lemieux, Allosteric regulation of rhomboid intramembrane proteolysis, EMBO J, 33 (2014) 1869-1881.

[31] Y. Xue, Y. Ha, Large lateral movement of transmembrane helix S5 is not required for substrate access to the active site of rhomboid intramembrane protease, J Biol Chem, 288 (2013) 16645-16654.

[32] M.K. Lemberg, J. Menendez, A. Misik, M. Garcia, C.M. Koth, M. Freeman, Mechanism of intramembrane proteolysis investigated with purified rhomboid proteases, EMBO J., 24 (2005) 464-472.

[33] K. Strisovsky, H.J. Sharpe, M. Freeman, Sequence-specific intramembrane proteolysis: identification of a recognition motif in rhomboid substrates, Mol. Cell, 36 (2009) 1048-1059. 
[34] S. Zoll, S. Stanchev, J. Began, J. Skerle, M. Lepsik, L. Peclinovska, P. Majer, K. Strisovsky, Substrate binding and specificity of rhomboid intramembrane protease revealed by substrate-peptide complex structures, EMBO J, 33 (2014) 2408-2421.

[35] E.V. Wolf, A. Zeissler, O. Vosyka, E. Zeiler, S. Sieber, S.H. Verhelst, A new class of rhomboid protease inhibitors discovered by activity-based fluorescence polarization, PLoS One, 8 (2013) e72307.

[36] J.M. Antos, G.L. Chew, C.P. Guimaraes, N.C. Yoder, G.M. Grotenbreg, M.W. Popp, H.L. Ploegh, Site-specific N- and C-terminal labeling of a single polypeptide using sortases of different specificity, J Am Chem Soc, 131 (2009) 10800-10801.

[37] O. Vosyka, K.R. Vinothkumar, E.V. Wolf, A.J. Brouwer, R.M. Liskamp, S.H.L. Verhelst, Activitybased probes for rhomboid proteases discovered in a mass spectrometry-based assay, Proc Natl Acad Sci USA, 110 (2013) 2472-2477.

[38] S.S. Twining, Fluorescein isothiocyanate-labeled casein assay for proteolytic enzymes, Anal Biochem, 143 (1984) 30-34.

[39] L.J. Jones, R.H. Upson, R.P. Haugland, N. Panchuk-Voloshina, M. Zhou, R.P. Haugland, Quenched BODIPY dye-labeled casein substrates for the assay of protease activity by direct fluorescence measurement, Anal Biochem, 251 (1997) 144-152.

[40] Y. Wang, Y. Zhang, Y. Ha, Crystal structure of a rhomboid family intramembrane protease, Nature, 444 (2006) 179-180.

[41] Y. Xue, Y. Ha, Catalytic mechanism of rhomboid protease GlpG probed by 3,4-dichloroisocoumarin and diisopropyl fluorophosphonate, J Biol Chem, 287 (2012) 3099-3107.

[42] C. Lazareno-Saez, E. Arutyunova, N. Coquelle, M.J. Lemieux, Domain swapping in the cytoplasmic domain of the Escherichia coli rhomboid protease, J Mol Biol, 425 (2013) 1127-1142.

[43] O.A. Pierrat, K. Strisovsky, Y. Christova, J. Large, K. Ansell, N. Bouloc, E. Smiljanic, M. Freeman, Monocyclic beta-lactams are selective, mechanism-based inhibitors of rhomboid intramembrane proteases, ACS Chem Biol, 6 (2011) 325-335.

[44] S.W. Dickey, R.P. Baker, S. Cho, S. Urban, Proteolysis inside the membrane is a rate-governed reaction not driven by substrate affinity, Cell, 155 (2013) 1270-1281.

[45] A.R. Sherratt, D.R. Blais, H. Ghasriani, J.P. Pezacki, N.K. Goto, Activity-Based Protein Profiling of the Escherichia coli GlpG Rhomboid Protein Delineates the Catalytic Core, Biochemistry, 51 (2012) 7794-7803.

[46] E.V. Wolf, M. Seybold, R. Hadravova, K. Strisovsky, S.H.L. Verhelst, Activity-based protein profiling of rhomboid proteases in liposomes, ChemBioChem, (2015) accepted.

[47] D.V. Jeyaraju, H.M. McBride, R.B. Hill, L. Pellegrini, Structural and mechanistic basis of Parl activity and regulation, Cell Death Differ, 18 (2011) 1531-1539. 
[48] S. Sekine, Y. Kanamaru, M. Koike, A. Nishihara, M. Okada, H. Kinoshita, M. Kamiyama, J. Maruyama, Y. Uchiyama, N. Ishihara, K. Takeda, H. Ichijo, Rhomboid protease PARL mediates the mitochondrial membrane potential loss-induced cleavage of PGAM5, J Biol Chem, 287 (2012) 3463534645.

[49] T.L. Cheng, Y.T. Wu, H.Y. Lin, F.C. Hsu, S.K. Liu, B.I. Chang, W.S. Chen, C.H. Lai, G.Y. Shi, H.L. Wu, Functions of rhomboid family protease RHBDL2 and thrombomodulin in wound healing, J Invest Dermatol, 131 (2011) 2486-2494.

[50] J.W. Harper, K. Hemmi, J.C. Powers, Reaction Of Serine Proteases With Substituted Isocoumarins Discovery Of 3,4-Dichloroisocoumarin, A New General Mechanism Based Serine Protease Inhibitor, Biochemistry, 24 (1985) 1831-1841.

[51] K.R. Vinothkumar, K. Strisovsky, A. Andreeva, Y. Christova, S. Verhelst, M. Freeman, The structural basis for catalysis and substrate specificity of a rhomboid protease, EMBO J, 29 (2010) 37973809.

[52] J. Vijayalakshmi, E.F. Meyer, C.M. Kam, J.C. Powers, Structural Study Of Porcine Pancreatic Elastase Complexed With 7-Amino-3-(2-Bromoethoxy)-4-Chloroisocoumarin As A Nonreactivatable Doubly Covalent Enzyme-Inhibitor Complex, Biochemistry, 30 (1991) 2175-2183.

[53] K.R. Vinothkumar, O.A. Pierrat, J.M. Large, M. Freeman, Structure of rhomboid protease in complex with beta-lactam inhibitors defines the S2' cavity, Structure, 21 (2013) 1051-1058.

[54] J.C. Powers, J.L. Asgian, O.D. Ekici, K.E. James, Irreversible inhibitors of serine, cysteine, and threonine proteases, Chem Rev, 102 (2002) 4639-4750.

[55] U. Haedke, E.V. Kuttler, O. Vosyka, Y. Yang, S.H.L. Verhelst, Tuning probe selectivity for chemical proteomics applications, Curr Opin Chem Biol, 17 (2013) 102-109.

[56] S. Serim, U. Haedke, S.H.L. Verhelst, Activity-based probes for the study of proteases: recent advances and developments, ChemMedChem, 7 (2012) 1146-1159.

[57] L.E. Sanman, M. Bogyo, Activity-based profiling of proteases, Annu Rev Biochem, 83 (2014) 249273.

[58] Y.S. Liu, M.P. Patricelli, B.F. Cravatt, Activity-based protein profiling: The serine hydrolases, Proc Natl Acad Sci USA, 96 (1999) 14694-14699.

[59] D. Kidd, Y.S. Liu, B.F. Cravatt, Profiling serine hydrolase activities in complex proteomes, Biochemistry, 40 (2001) 4005-4015.

[60] D.A. Bachovchin, S.J. Brown, H. Rosen, B.F. Cravatt, Identification of selective inhibitors of uncharacterized enzymes by high-throughput screening with fluorescent activity-based probes, Nat. Biotechnol., 27 (2009) 387-394. 
[61] Z. Wu, N. Yan, L. Feng, A. Oberstein, H. Yan, R.P. Baker, L. Gu, P.D. Jeffrey, S. Urban, Y. Shi, Structural analysis of a rhomboid family intramembrane protease reveals a gating mechanism for substrate entry, Nat Struct Mol Biol, 13 (2006) 1084-1091.

[62] A. Ben-Shem, D. Fass, E. Bibi, Structural basis for intramembrane proteolysis by rhomboid serine proteases, Proc Natl Acad Sci USA, 104 (2007) 462-466.

[63] M.J. Lemieux, S.J. Fischer, M.M. Cherney, K.S. Bateman, M.N. James, The crystal structure of the rhomboid peptidase from Haemophilus influenzae provides insight into intramembrane proteolysis, Proc Natl Acad Sci USA, 104 (2007) 750-754.

[64] R.P. Baker, K. Young, L. Feng, Y. Shi, S. Urban, Enzymatic analysis of a rhomboid intramembrane protease implicates transmembrane helix 5 as the lateral substrate gate, Proc Natl Acad Sci USA, 104 (2007) 8257-8262.

[65] Y. Xue, S. Chowdhury, X. Liu, Y. Akiyama, J. Ellman, Y. Ha, Conformational change in rhomboid protease GlpG induced by inhibitor binding to its S' subsites, Biochemistry, 51 (2012) 3723-3731.

[66] R.P. Baker, S. Urban, Cytosolic extensions directly regulate a rhomboid protease by modulating substrate gating, Nature, (2015).

[67] Y. Zhou, S.M. Moin, S. Urban, Y. Zhang, An internal water-retention site in the rhomboid intramembrane protease GlpG ensures catalytic efficiency, Structure, 20 (2012) 1255-1263.

[68] T. Bottcher, S.A. Sieber, beta-lactones as privileged structures for the active-site labeling of versatile bacterial enzyme classes, Angew Chem Int Ed, 47 (2008) 4600-4603.

[69] P. Kasperkiewicz, M. Poreba, S.J. Snipas, H. Parker, C.C. Winterbourn, G.S. Salvesen, M. Drag, Design of ultrasensitive probes for human neutrophil elastase through hybrid combinatorial substrate library profiling, Proc Natl Acad Sci USA, 111 (2014) 2518-2523.

[70] T. Reddy, J.K. Rainey, Multifaceted substrate capture scheme of a rhomboid protease, J Phys Chem B, 116 (2012) 8942-8954.

[71] L. Fleig, N. Bergbold, P. Sahasrabudhe, B. Geiger, L. Kaltak, M.K. Lemberg, Ubiquitin-dependent intramembrane rhomboid protease promotes ERAD of membrane proteins, Mol. Cell, 47 (2012) 558-569. [72] J. Singh, R.C. Petter, T.A. Baillie, A. Whitty, The resurgence of covalent drugs, Nat Rev Drug Discov, 10 (2011) 307-317.

[73] R.A. Bauer, Covalent inhibitors in drug discovery: from accidental discoveries to avoided liabilities and designed therapies, Drug Discov Today, (2015). 